\title{
Sepsis and septic encephalopathy: characteristics and experimental models
}

\author{
Marek Ziaja \\ Department of Neuroanatomy, Institute of Zoology, Jagiellonian University, Kraków \\ ${ }^{*}$ Current affiliation: Department of Histology, Jagiellonian University Medical College, Kraków
}

\begin{abstract}
The inflammation is a response of the organism to damaging factors and leads to the limitation of the tissue destruction. During the inflammatory process, there is stimulation of the immune system as well as other tissue cells. However, sometimes this reaction is excessive and can bring to the sepsis and development of multiorgan insufficiency. Phenomena observed during sepsis influence also, directly or indirectly on the nervous system and cause septic encephalopathy (SE) with consciousness and cognitive functions loss and other neurological symptoms. Often it can lead to persistent brain injuries, and almost always cause changes, which can be manifested later, even many years after the sepsis. It is supposed that in many cases, septic encephalopathy can be the main reason of death during sepsis. Still increasing concern of SE brought to the development of several animal models of this syndrome, which made possible detailed recognition of phenomena accompanying of septic encephalopathy. They include direct administration of endotoxins, or surgical intervention within the abdominal cavity. Every presented experimental model has advantages and weakness, but they make possible the modeling of the inflammatory reaction and multidirectional examining of accompanying phenomena.
\end{abstract}

Key words: sepsis, encephalopathy, inflammation, endotoxin, model.

\section{Inflammation}

Inflammation is the specific response of blood containing tissues to damaging factors. The purpose of this complex process when different cell types participate is the neutralization and removal of the causative factor and repair of damaged tissues. The local reaction is usually accompanied by generalized symptoms such as the fever, metabolic or hormonal disorder, and can lead to the shock. Inflammation can have a severe or chronic form, with similar patterns but with a different duration, and sometimes the chronic form can be a consequence of the severe one or an idiopathic process.

When the damaging factor affects tissue, first changes are observed in the microcirculation, where after the initial, short-term counteraction, relaxation of vessels and an enlarged influx of blood to the damaged area follow. Simultaneously, the permeability of vessels increases, what causes the outflow of pro- 
teins from the blood plasma to neighbouring tissues. On the surface of endothelial cells the expression of adhesion molecules increases and enables the influx of leukocytes into the inflammation focus. In the following sequence, within the damaged area macrophages remove debris. Further, the resorption of the effusion occurs and lastly, the new vascular network in the tissue is formed. The homeostasis is restored when the inflammation is limited by the quick and intensive anti-inflammatory reaction, which is reversible, limited to the damaged structure and adapting to changes throughout the inflammatory reaction.

The inflammation has a similar character of the reaction regardless of the sort of the damaging factor (specific or non-specific), and is also characterised by a simultaneous occurrence of adversative phenomena (e.g. simultaneous expression of pro- and antiinflammatory cytokines) [25]. This process is controlled by inflammatory mediators with complicated activity because different mediators can cause an analogous result, the same mediator can make dissimilar effects, or the synergistic activity of several mediators can happen.

The intensity of the inflammatory reaction is crucial, because an insufficient response can lead to the immunodeficiency which can favour infections or the carcinogenesis. On the other hand, overactivity may be the reason of increased morbidity and mortality in different diseases such as rheumatoid arthritis, Alzheimer's disease, multiple sclerosis or cerebral ischemia $[9,25]$. If inflammatory mediators expand to the blood stream, what can happen e.g. in the septic shock, sepsis or meningitis, the response can be more dangerous than the straight factor causing this reaction.

\section{Sepsis}

Sepsis is characterized by the inflammatory reaction of the whole body and simultaneous activation of congenital anti-inflammatory mechanisms, and a balance among these processes is of the critical significance [23]. It is then defined as the Systemic Inflammatory Reaction Syndrome (SIRS) caused by the infection [8]. Despite the considerable progress in the diagnosing and treatment, there is a still high rate of mortality in the sepsis which is the most frequent cause of death among patients of intensive care units, and additionally the morbidity increases from $1.5 \%$ to $8 \%$ annually. Moreover, the overall mortality reaches from $30 \%$ to $70 \%$ of cases $[16,17]$.
The SIRS occurs when at least two of the symptoms mentioned below appear:

- hyper- or hypothermia - if the body temperature is above $38^{\circ} \mathrm{C}$ or below $36^{\circ} \mathrm{C}$,

- tachycardia - the pulse rate over 90 per minute,

- tachypnea - frequency of breaths above 20 per minute, or the value $\mathrm{PaCO}_{2}$ below $32 \mathrm{~mm} \mathrm{Hg}$,

- leukocytosis - number of white blood cells above 12000 per $\mathrm{mm}^{3}$, or

- leukopenia - number of white blood cells below 4000 per $\mathrm{mm}^{3}[11,12]$.

After the recognition of SIRS symptoms, it is necessary to define the causative pathogen. The most frequent factor causing sepsis is the infection (75-85\%) caused by both Gram-negative and Gram-positive bacteria $[11,12]$. The infection mainly spreads out through lungs, kidneys and urinary tracts and also abdominal organs [1]. It is interesting that in almost $2 / 3$ cases of the severe sepsis, the identification of the pathogen responsible for the infection failed. Therefore, the statement of the bacteria in the blood is not necessary to diagnose SIRS but if it occurred in the blood, it is so called bacteraemia. Except the bacterial infection, sepsis can be also caused by viruses as well as fungi [10]. Often the primary reason of the sepsis can be trauma, surgical treatment or burns, and also tumour diseases and pneumonia; persons with the compromised immunity and elderly people are most exposed.

The course of the sepsis is complicated and concerns the cellular as well as tissue level. In the early phase, both the cellular and humoral response of the immune system undergoes hyperactivation [11,12]. It manifests mainly by enhanced production and release of proinflammatory mediators such as TNF- $\alpha$ and interleukins: IL-1, IL-6, IL-8 by endothelial cells, neutrophils, macrophages and lymphocytes. Simultaneously soluble elements of the immune system are activated [10]. In the blood plasma the concentration of acute-phase proteins such as $C$ reactive protein increases and the cascade of the complement system leads to the appearance of such products as components $\mathrm{C} 3 \mathrm{a}$ or $\mathrm{C} 5 \mathrm{a}$ which additionally augment the synthesis of cytokines [11,12]. This enhanced production of cytokines appears about 12-24 hours after the infection and clinically reveals SIRS symptoms mentioned above. Furthermore, the coagulation cascade is quickly activated, which leads to the Disseminated Intravascular Coagulopathy (DIC) $[11,12]$. The main feature of the septic shock is blood circulation disturbances. 
It leads to dysfunction of the haemostatic equilibrium which causes thrombosis and bleeding. In physiological conditions the sensitive balance between coagulation and fibrinolysis is maintained. Due to the activation of the coagulation cascade, the thrombin converts fibrinogen to the fibrin, the final product of the hemostasis. In turn, the fibrinolytic system then acts towards the dissolution of the fibrinogen and the fibrin. Its activation causes production of the plasmin which in the presence of the thrombin brings about the dissolution of fibrin clots. Thus, what is critical in this equilibrium is the presence of the thrombin as the main proteolytic enzyme of the coagula- tion and simultaneously indispensable in the fibrinolysis [5].

In the septic shock, there is deprivation of the control mechanisms of coagulation and fibrinolysis, which results in formation of many clots and consequent bleeding (Fig. 1). One of the critical factors of these processes is the secretion of the tissue factor on the surface of distinct cells such as the endothelium, macrophages or monocytes pursuant to the vascular damage under the stimulation through inflammatory cytokines as IL-1, TNF- $\alpha$, and also endotoxins. It is essential that the tissue factor abundantly appears in different tissues as well as in the brain. Moreover, there is also produc-

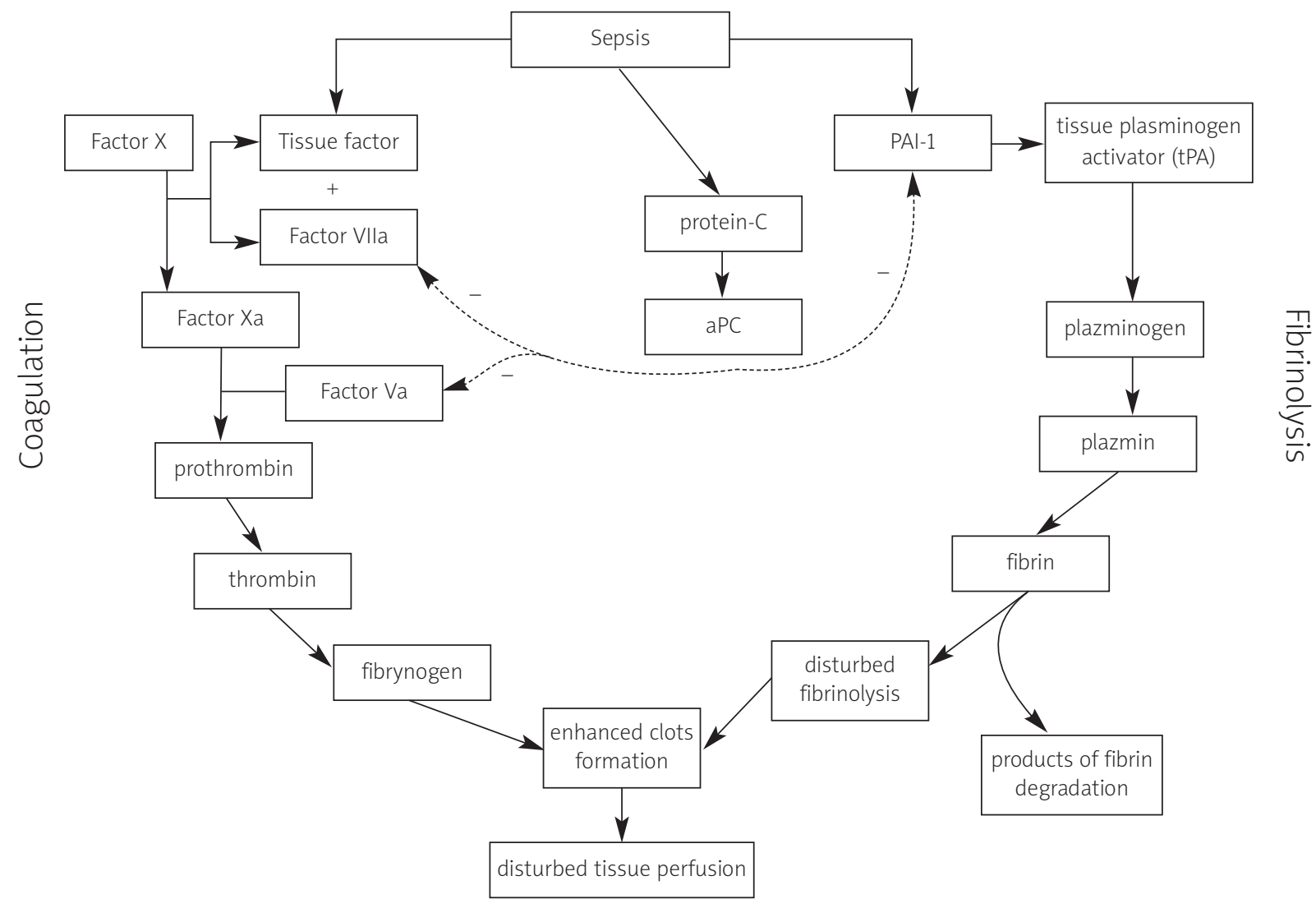

Fig. 1. During sepsis the expression of the tissue factor increases, what causes the growth of prothrombin production, which is converted to the thrombin. Thrombin generates the transformation of fibrinogen to fibrin. Simultaneously, the level of the plasminogen activator inhibitor-1 (PAI-1) increases, what leads to the diminution of the plasmin synthesis and normal fibrinolytic mechanism inhibition, whereby the fibrin is dissolved. During sepsis, the natural anticoagulation protein- $C$ level also decreases. Activated protein- $C$ (aPC) dissociates from the endothelial protein-C receptor, inactivates factors $\mathrm{Va}$ and VIla and inhibits the activity PAI-1. Therefore, a diminished level of protein- $C$ causes an additional enlargement of the coagulation. Disturbance of the coagulation and the anticoagulation mechanisms results in the enlarged production of clots of the fibrin in capillary vessels, what leads to perturbation of tissue's perfusion, and to a decreased supply of oxygen and finally the cell death. From Cohen, 2002, modified. 
tion of the plasminogen activator inhibitor 1 (PAI-1), which additionally impedes fibrinolysis. The excess of circulating thrombin arises from the extreme activation of the coagulation cascade. The thrombin cuts the fibrinogen which finally leaves many clots of the fibrin in blood vessels. These clots catch platelets enlarging their own size, what leads to the vascular thrombosis. The accumulation of such thrombosis leads to ischemia, perfusion disturbances and at last to the extreme destruction of the organ and decrease of the coagulation inhibitor level also favours this process. Simultaneously, the excess of circulating thrombin brings about the conversion of plasminogen to the plasmin and the fibrinolysis [5]. Catabolites of thromboses have a very strong antithrombotic activity what, on the other hand, causes numerous microhaemorrhages.

These processes lead to the state of hypercoagulation when hemorrhagic symptoms and multiorgan insufficiency occur [5]. Phagocytes (macrophages, neutrophils) in response to tissue destruction, release enzymes and generate reactive free radicals which take part in the bacteria elimination process. However, it simultaneously causes secondary tissue damage what finally leads to more increased blood vessel permeability and organ injury $[11,12]$.

The later stage of the sepsis, defined as the hypodynamic response phase [10] is characterized by strong production of anti-inflammatory factors, which can neutralize the activity of proinflammatory cytokines to restore the balance among both types of the immune response [10]. The enhanced production of IL-10, IL-13 and TGF- $\beta$ is noted $[16,17]$ and so is a significant fall of the phagocytic activity of macrophages and neutrophils, decrease of their antibacterial capabilities, the diminution in production of reactive oxygen species, cytokines and also decline of the chemotaxis ability. Mechanisms leading to this type of immunosuppression still did not become clearly recognized but that immune response inhibition favours consecutive infections [10].

Unless proper effective treatment is quickly applied, sepsis can be accompanied by the Multi Organ Dysfunction Syndrome (MODS), in which intravascular dissemination and disturbances of at least two organs or systems can be observed. MODS may affect the nervous system, blood circulation, urinary and digestive systems and liver. The multiorgan insufficiency is the basic problem in the sepsis treatment because it appears in $30 \%$ of patients and constitutes the major cause of death [11]. MODS can also develop as a result of the septic shock which appears in about 20-50\% of patients with sepsis [16] and occurs at the moment of exhaustion of compensatory mechanisms of the patient. It is defined as the systemic inflammatory reaction caused by the infection in which, contrary to the other forms of the shock, hypotension and the insufficient organs' perfusion remain in spite of the proper filling of the vasculature [19]. The septic shock is characterized by broad changes in the heartvascular system [7]. Consequently, these changes lead to tissue hypoperfusion which in turn contributes to the appearance of MODS. Therefore, the septic shock is a state in which insufficient quantities of oxygen and nutrients are provided to the tissues and leads to the lactate acidosis and the hypoxia. If this state is longlasting, an irreversible damage of tissues and organs appear and finally leads to death [7] (Fig. 2).

\section{Septic encephalopathy}

One of the organs which are strongly affected by the activity of inflammatory processes in the septic shock is the brain. The central nervous system is considered as at least partly "immunologically privileged" as regards the occurrence of the specialized bloodbrain barrier, structurally and functionally separating the nerve tissue from the influence of blood-borne factors. Moreover, the lack of the classical lymphatic system, and the expression of major histocompatibility complex class II appear on a very low level. Besides, endothelial cells of cerebral vessels have a diminished expression of adhesion molecules such as VCAM-1 and ICAM-1, what greatly impedes the adhesion of leucocytes circulating in blood [22]. Nonetheless, also in the brain there are immune processes, happening both under changes in the brain itself and as well as being an effect of the influx of inflammatory factors from blood across the blood-brain barrier.

The inflammatory response in the brain can be initiated by endogenous factors such as embolism of blood vessels leading to the ischemia and the hypoxia or neoplastic changes, or exogenous factors such as bacteria and viruses, mechanical injuries, or chemical substances. In each such case, there is an activation of not only immune system cells but also endogenous ones, mainly astrocytes and microglia.

Distempers of brain functionality can be an effect of pathological changes of other body organs e.g. the liver. Such phenomenon is defined as encephalopa- 
thy, described as the decrease of the mental state or altered consciousness caused by morbid processes developed outside the brain $[12,14]$. It is considered not as a separate disease entity but rather as a syndrome of the general brain dysfunction caused by different factors. It can be caused by infectious factors such as bacteria or viruses, metabolic disorders, exposure to toxins, radiation, injury, disturbances of the blood flow, etc. Main features of the encephalopathies are consciousness disorders, and depending on the type and severity of encephalopathy, common neurological symptoms are the loss of cognitive functions, subtle personality changes, lack of concentration and depression $[2,5,6]$. Other symptoms can be myoclonus, asterixis, tremor, epileptic seizures or respiratory dysfunction [6]. In some cases, it comes to permanent mental injuries as in the case of the septic encephalopathy, while in other cases, these changes can be reversible as in the case of encephalopathies caused by toxins or nutritional failure.

In the majority of types of encephalopathies, there is a consciousness dysfunction due to the release and the later intensification of the inflammatory reaction. The most distinct example is the septic encephalopathy, being developed in the patient during sepsis and/or the septic shock and is the most frequent form of encephalopathy. The patient with sepsis often has characteristic symptoms of the encephalopathy, such as anxiety, excitableness, stupor, and even the coma [14]. It is estimated that in intensive care units, encephalopathy affects even up to $71 \%$ of septic shock patients. It appears that also mortality among patients with ascertained encephalopathy is distinctly greater than in these in whom such symptoms have not been discovered [14].

The proper diagnosis of the encephalopathy signs is of key importance, because patients, before their state of mind is examined, are often given strong anaesthetics, which mask neurological disorders whose effects appear sometimes many years after the past illness. This happens because the mental injury in the progress of septic associated encephalopathy (SAE) can be reversible. However, in many cases a permanent decrement of the brain tissue can appear. As yet there is no definite and effective method of the SAE treatment and favourable results are attained thanks to the suitable therapy of the sepsis itself.

Nevertheless, bacterial infections seem to be the most frequent reason of sepsis, in the vast major-

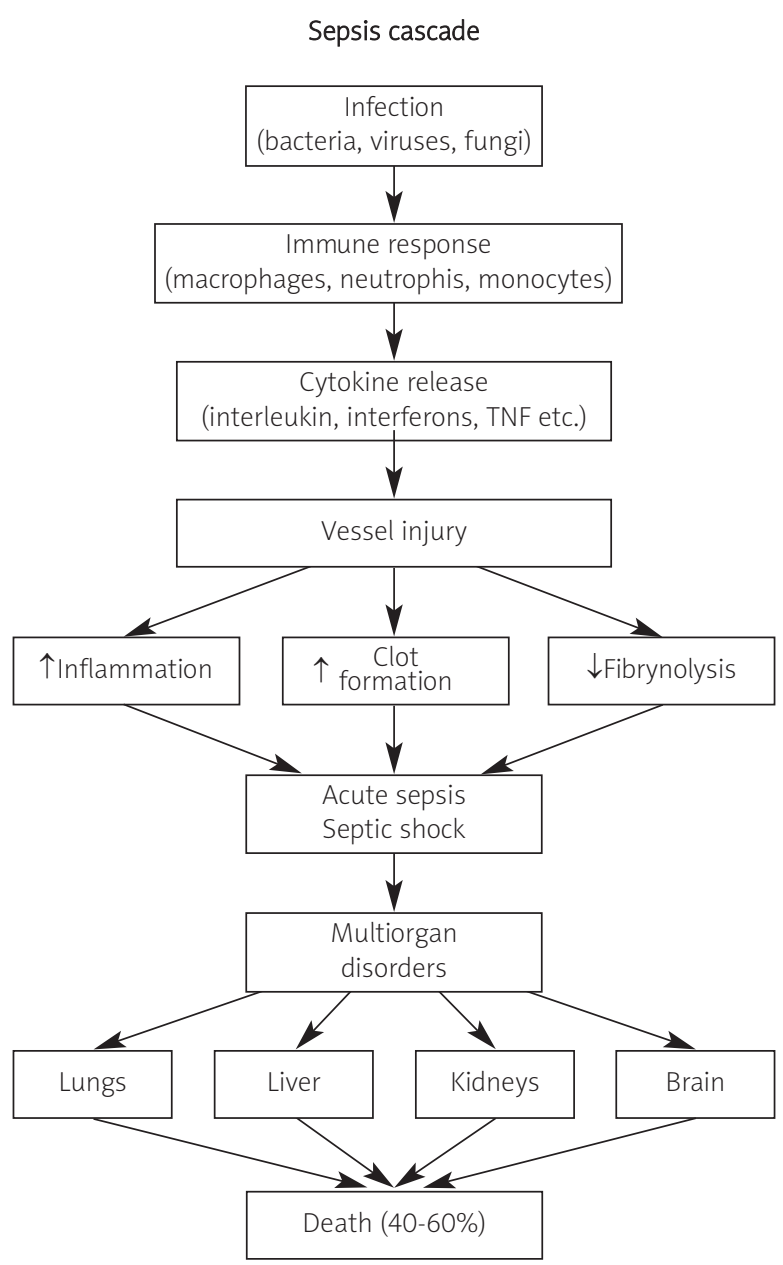

Fig. 2. The order of phenomena observed in the progress of the sepsis. Infection causes stimulation of the immune-system cells which secrete active compounds. Destabilization of the inflammatory and anti-inflammatory response equilibrium leads to intensification of the proinflammatory factors' secretion, what causes further increase of the inflammatory reaction. This augmentation disturbs the equilibrium between coagulation and anticoagulation, what results in the excessive production of clots and inhibition of the proper perfusion of tissues. In consequence, the septic shock develops and brings about multiorgan disorders and in effect, approximately $40 \%$ to $60 \%$ of patients die.

ity of septic encephalopathy cases any bacteria occurrence could not be ascertained in the nervous system. Therefore, it is rather improbable that microorganisms 
in the brain would directly cause septic encephalopathy. Hence, to underline lack of the direct infection of the nervous system, the term Sepsis Associated Encephalopathy was introduced to define the disperse brain dysfunction caused by the overall body reaction to infection without any clinical or laboratory evidence of direct infection [6].

The SAE aetiology is unclear and many mechanisms of the development of the septic encephalopathy are proposed [8]. Factors which can be involved in its development include metabolic changes and anomalies within the cerebral circulation [7], increase of cytokines and proinflammatory concentration, changes in the permeability of the blood-brain barrier, damage of endothelial cells [4], changes in the level of neurotransmitters in the brain and amino acids in plasma [8]. The direct influence of the bacteria or their toxins on the brain is also taken into consideration [8]. Practically, the brain dysfunction during sepsis can be linked with the activity of all components mentioned above. Therefore, the SAE pathogenesis is most likely multifactorial [14].

It is known that endotoxins of the bacterial origin such as e.g. lipopolysaccharide (LPS) are factors considerably stimulating inflammatory reaction, and they are also observed in the progress of SAE. Lipopolysaccharide, a component of the wall of Gram-negative bacteria has been used as a stimulant of inflammation for many years. This compound consists of three structural parts, O-chain, core region and lipid-A and this last one is responsible for LPS toxicity. In aqueous solutions, LPS particles aggregate, what results in its biological inactivation [26]. LPS activity is attained during monomerisation [19] through LPS binding proteins (LBP) circulating in blood which concentration increases considerably during inflammation [16,19]. It is interesting that LBP is also responsible for LPS detoxification through enzymatic connection with high-densitylipoprotein (HDL), what deactivates LPS [26]. Thus, the LBP occurrence can indicate different, opposed phenomena in the progress of the inflammatory process. The activation of the immune-system cells occurs through LPS-LBP complex connection with mCD14 receptor appearing on the outer membrane of neutrophils and monocytes/macrophages. In the brain, this type of receptors exists on microglial cell's surfaces so they can actively react on bacterial toxins in the tissue. Endothelial and smooth muscle's cells do not possess CD14 protein and they are stimulated by soluble CD14 (sCD14) circulating in the blood and secreted by immune-system cells. An active complex of LPS-LBPCD14 arises, which, through Toll-like receptors, mainly type 2 and 4 , stimulates synthesis of suitable proinflammatory cytokines [16,19]. Alternative activation without formation of LPS-LBP-CD14 complexes is also possible and this happens at very large LPS concentrations in blood, and the mechanism is not fully recognized. However, and in this case, LPS shows the activity only in the form of the monomer.

LPS activated cells secrete a variety of inflammatory cytokines such as IL-1, IL-6, and TNF- $\alpha$. IL-1 and TNF- $\alpha$ are secreted within 30-90 minutes from the LPS delivery and activate the second stage of the inflammatory response, i.e. synthesis of other factors such as other cytokines, reactive oxygenic radicals, the nitric oxide and increase the expression of adhesion molecules, which results in the beginning of an inflammatory cells influx to the tissue and further propagation of the response $[16,19]$. The enlarged proinflammatory response is balanced by a series of regulatory molecules, which restore immune equilibrium $[15,16]$. In this meaning anti-inflammatory response appears as a modifier of the inflammatory reaction. Active substances such as soluble TNF receptors and receptor IL-1 antagonists, "trapping" receptors such as type II receptors of IL-1, inactivators of the complement cascade and anti-inflammatory cytokines such as IL-10, IL-14, TGF- $\beta$ are secreted $[15,16]$. At the same time, there are changes of the metabolic activity such as amplified production of the cortisol and catecholamines, induction of acute-phase proteins and endothelium activation with an increased expression of adhesion molecules, prostanoids and platelet-activating factor (PAF) secretion. Other factors weakening the immune response is apoptosis of lymphocytes, which can be observed in the animal models as well as in sepsis patients. Studies show that it leads to selective diminishing of lymphocytes B and Th pool [5].

Direct crossing of endotoxins by the unimpaired blood-brain barrier is difficult, so they probably exert an indirect influence through stimulating the secretion of inflammatory substances. They increase the amount of free radicals which cross BBB and have a destroying influence on endothelial cells, astrocytes and neurons, what in turn leads to distemper of the brain function $[6,14]$. Moreover, the antioxidant system, protecting the cells from an influence of free radicals, stops to act properly, which is testified by an obviously lower concentration of the ascorbic acid in the cerebrospinal 
fluid and blood plasma of patients with septic encephalopathy. Besides, changes of its concentration are clearly correlated with the intensity of neurological dysfunctions throughout the septic encephalopathy. Furthermore, endotoxins disturb the hypothalamus-pituitary hormonal axis. In SAE, destruction of BBB is usually observed although glial cells try to increase their own activity to maintain BBB coherence. Disestablishment of the barrier integrality leads to acute disturbances in the influx of many compounds whose blood plasma and CSF concentration undergoes dramatic changes. In this respect, it leads to disturbances of quantitative relations of aromatic and branched-chain amino acids, what in turn induces the reduction of the cerebral concentration of norepinephrine, dopamine and serotonin, but the GABA level remains unaltered [6]. Therefore, distempers of the state of mind in SAE are credited to the excess of aromatic amino acids, especially that described dysfunctions were observed already 12 hours after the appearance of SAE symptoms [2].

During the multiorgan inflammatory state, most frequent damages of the brain are located in the cerebral cortex of the telencephalon, but sometimes also in deep structures and even in the spinal cord [6]. Most predominant changes in the brain are ischaemic lesions in different structures, and especially in nuclei of the autonomic nervous system. Frequently observed signs of apoptosis are not strictly correlated with changes caused by ischemia [15] and accompanied by perivascular swelling and destruction of astrocytic endfeets, damaged neurons have shrunken nuclei and a destroyed cell membrane [15]. Because micro abscesses in the tissue were found very seldom, these changes could not be the direct effect of infection. Moreover, intracerebral hemorrhagic lesions, similar to necrotic foci detected in the brains of people who died because of disseminated intravascular coagulopathy, were found in rat brains following an endotoxin injection [3]. There were also found enlargements around endothelial cells, containing macrophages and perivascular accumulations of erythrocytes, especially in the frontal lobe, and also necrosis foci in the white matter [24]. Furthermore, microglia and astrocyte increase their metabolic activity [21].

\section{Methods of the systemic inflammation model development}

Biological mechanisms of the disease have become known using in vitro models, which enable the maxi- mum control of conditions of the experiment and minimize the liability and formation of false data. However, there are in vivo animal models that permit verifying various hypotheses. The main goal of all models of the systemic inflammatory reaction is to reflect most faithfully the pathogenesis of sickness-changes observed in people. At present, several principal models to develop inflammatory changes in experimental animals are elaborated on.

Endotoxemia. This is the simplest, most often used and repeatable method of the induction of inflammatory changes. It arises from the assumption that a factor causing the inflammatory reaction is not a bacterium itself, but bacterial toxins. The name endotoxin is sometimes used alternatively with LPS, because LPS is the main biologically active compound of the endotoxin. The administration of LPS intravenously or intraperitoneally cause similar symptoms as in sepsis such as e.g. dysfunction of the blood vascular system. Besides, LPS application induces the increase of the concentration of proinflammatory cytokines in the blood serum, similarly as in septic patients. However, this phenomenon has a subtly different profile because the levels of inflammatory cytokines (TNF- $\alpha$, IL-6, CXC, chemokines) are much higher in this model and attain the maximum earlier than it is observed in patients with sepsis. There are adapted different variants of the endotoxemia model: single, sublethal intravenous small doses of endotoxin, a continuous endovenous delivery of LPS little doses and the administration of LPS intraperitoneally. This model has advantages as well as disadvantages. LPS is a stable compound of well-known origin, easy to store, which gives a possibility of single or repeated delivery of equal doses of the endotoxin, and make the method very repeatable. Furthermore, it is simple to change the quantity of LPS given and to modulate the response of the organism to endotoxin and more simply to observe chosen life parameters during the inflammatory reaction. On the other hand, it is necessary to remember about several limitations of this method. A single dose of the endotoxin can insufficiently reflect the immune response in humans. Depending on the dose and the way of administration it is possible to receive a different hemodynamic response and that there can be species-specific discrepancies in response to LPS. The liability of the results obtained with that method of sepsis developing can originate from several sources such as the type of toxin used, the dose 
lethality, the way of application, etc. and lastly, there is no focus of the infection in the organism. However, since this method can be modified, there is an opportunity to change examined vital parameters, so this is one of the most often used methods of induction of inflammatory changes. What is interesting, this method is sometimes applied in volunteers with the use of very small doses of LPS and subsequent studying of hemodynamic parameters $[23,25,26]$.

Inoculation with bacteria. An alternative method of creating septic changes is the administration of live bacteria. It can be made in two ways, i.e. either through inoculation with the precise dose of the bacteria of the given breed, or the intraperitoneal implantation of faeces particles, while the latter reminds partly cecal ligation and puncture (CLP - described below) and at present was replaced by later/by the latter. In the case of administration of live bacteria there can be several difficulties. It is essential to culture a particular bacteria breed and administrate them in very large doses, because a considerable part is removed from the organism by the immune system. Moreover, as a consequence it can give enormous differences among various research centres. Besides, the response of the host is related to the genus of bacteria used, places and time of their administration and sometimes genetic features of the host being subjected to bacterial inoculation influence the response to the infection with a given pathogen. On the other hand, it is possible to select a suitable breed of infecting bacteria and in adequately controlled conditions verify their quantity in a definite dose. In some cases, bacteria are injected together with adjuvant to increase the activity of immune-system cells. However, the administration of homogenous bacterium culture reminds a model of the endotoxemia rather than peritonitis. At present, it is considered that both these models reflect more the occurrence of abscesses in the abdominal cavity as a result of the peritonitis than the sepsis beginning in the abdominal cavity. Besides, they are more useful to examine the mechanism of the host response to the occurrence of particular pathogens. An advantage of this method is the possibility of causing infections of determined organs such as lungs (bronchial application) or the kidney $[4,13,18,20]$.

Cecal Ligation and Puncture model (CLP) is considered as a standard procedure in sepsis research, because it imitates cases of the human appendicitis connected with appendix perforation and peritonitis. CLP relies on the laparoscopic method of extraction of the appendix and its ligation and perforation. This treatment causes leakage of the faecal content from the intestine to the peritoneum, what causes infection with the different bacterial flora and creates the inflammatory source of the necrotic tissue. The severity of the disease, measured by mortality, can be modulated due to the change of the needle diameter used for the perforation and the quantity of punctures to the appendix so that the death follows within several hours or even days. It is interesting to note that CLP reproduces hemodynamic and metabolic changes developing during the human sepsis. Moreover, the apoptosis of various cell types and the immune response seem to imitate the course of the human disease. There are, however, factors influencing results received in this method. One of them is the size of appendix, what is the main determinant of the mortality, because together with the increase of its length, the quantity of proinflammatory cytokines like IL- 6 and TNF- $\alpha$ significantly grows. This is why it is indispensable to maintain highest standards during the treatment and later on $[23,25]$. Recently, a new model which partly eliminates reasons of the liability of the inflammatory reaction after CLP has been developed. This is so called colon ascendens stent peritonitis (CASP) $[4,18]$. The modification is as follows: a drainpipe is inserted in the descending part of the large intestine, over the ileo-cecal valve and allows for the outflow of the content of the intestine to the peritoneal space, what causes peritonitis. An advantage of this method is the fact that after a determined time, the drainpipe can be removed and the entire procedure imitates the surgical intervention. Experiments on mice indicate, however, that positive results were obtained as far as a drainpipe was removed within first 3 hours after the implantation, and its later removal does not cause the recovery of the mouse. Usually animals die because of multiorgan damages, e.g. in lungs, kidneys and liver as normally are seen in the human multiple organ dysfunction syndrome. Nevertheless, CLP still remains "a golden standard" in research of the sepsis and septic shock $[4,13,18,20]$.

\section{References}

1. Astiz ME, Rackow EC. Septic shock. Lancet 1998; 351: 1501-1505.

2. Basler T, Meier-Hellmann A, Bredle D, Reinhart K. Amino acid imbalance early in septic encephalopathy. Intensive Care Med 2002; 28: 293-298.

3. Bogdanski R, Blobner M, Becker I, Hanel F, Fink H, Kochs E. Cerebral histopathology following portal venous infusion of bacteria in a chronic porcine model. Anesthesiology 2000; 93: 793-804. 
4. Buras JA, Holzmann B, Sitkovsky M. Animal models of sepsis: setting the stage. Nat Rev Drug Discov 2005; 4: 854-865.

5. Cohen J. The immunopathogenesis of sepsis. Nature 2002; 420: 885-891.

6. Consales G, De Gaudio AR. Sepsis associated encephalopathy. Minerva Anestesiol 2005; 71: 39-52.

7. De Backer D. Hemodynamic management of septic shock. Curr Infect Dis Rep 2006; 8: 366-372.

8. Green R, Scott LK, Minagar A, Conrad S. Sepsis associated encephalopathy (SAE): a review. Front Biosci 2004; 9: 1637-1641.

9. Guo MF, Yu JZ, Ma CG. Mechanisms related to neuron injury and death in cerebral hypoxic ischaemia. Folia Neuropathol 2011; 49: 78-87.

10. Hoesel LM, Gao H, Ward PA. New insights into cellular mechanisms during sepsis. Immunol Res 2006; 34: 133-141.

11. Karima R, Matsumoto S, Higashi H, Matsushima K. The molecular pathogenesis of endotoxic shock and organ failure. Mol Med Today 1999; 5: 123-132.

12. Kunze K. Metabolic encephalopathies. J Neurol 2002; 249: 1150-1159.

13. Marshall JC, Deitch E, Moldawer LL, Opal S, Redl H, van der Poll T. Preclinical models of shock and sepsis: what can they tell us? Shock 2005; 24 Suppl 1: 1-6.

14. Papadopoulos MC, Davies DC, Moss RF, Tighe D, Bennett ED. Pathophysiology of septic encephalopathy: a review. Crit Care Med 2000; 28: 3019-3024.

15. Papadopoulos MC, Lamb FJ, Moss RF, Davies DC, Tighe D, Bennett ED. Faecal peritonitis causes oedema and neuronal injury in pig cerebral cortex. Clin Sci (Lond) 1999; 96: 461-466.

16. Riedemann NC, Guo RF, Ward PA. The enigma of sepsis. J Clin Invest 2003; 112: 460-467.

17. Riedemann NC, Guo RF, Ward PA. Novel strategies for the treatment of sepsis. Nat Med 2003; 9: 517-524.

18. Rittirsch D, Hoesel LM, Ward PA. The disconnect between animal models of sepsis and human sepsis. J Leukoc Biol 2007; 81: 137-143.

19. Rowe PM. Septic shock: finding the way through the maze. Lancet 1999; 354: 2058.

20. Schultz MJ, van der Poll T. Animal and human models for sepsis. Ann Med 2002; 34: 573-581.

21. Sharshar T, Gray F, Poron F, Raphael JC, Gajdos P, Annane D. Multifocal necrotizing leukoencephalopathy in septic shock. Crit Care Med 2002; 30: 2371-2375.

22. Streck EL, Comim CM, Barichello T, Quevedo J. The septic brain. Neurochem Res 2008; 33: 2171-2177.

23. Takala A, Nupponen I, Kylanpaa-Back ML, Repo H. Markers of inflammation in sepsis. Ann Med 2002; 34: 614-623.

24. Tamada F. Brain injury induced by continuous infusion of endotoxin in rats-protective effects of methylprednisolone on intracerebral blood vessels. No To Shinkei 1993; 45: 49-56.

25. Tracey KJ. The inflammatory reflex. Nature 2002; 420: 853-859.

26. Woltmann A, Hamann L, Ulmer AJ, Gerdes J, Bruch HP, Rietschel ET. Molecular mechanisms of sepsis. Langenbecks Arch Surg 1998; 383: 2-10. 